\title{
Review \\ Plant Immunity: From Signaling to Epigenetic Control of Defense
}

\author{
Juan S. Ramirez-Prado, , ,2,4 Aala A. Abulfaraj, ${ }^{1,3,4}$ Naganand Rayapuram,, ${ }^{1,4}$ Moussa Benhamed, ${ }^{2, *}$
} and Heribert Hirt ${ }^{1,2, *}$

Pathogen recognition by plants results in the activation of signaling pathways that induce defense reactions. There is growing evidence indicating that epigenetic mechanisms directly participate in plant immune memory. Here, we discuss current knowledge of diverse epigenomic processes and elements, such as noncoding RNAs, DNA and RNA methylation, histone post-translational modifications, and chromatin remodeling, that have been associated with the regulation of immune responses in plants. Furthermore, we discuss the currently limited evidence of transgenerational inheritance of pathogeninduced defense priming, together with its potentials, challenges, and limitations for crop improvement and biotechnological applications.

Immunity, Defense, and Memory

In their natural environment, plants coexist in an intimate relationship with highly dynamic microbial communities. As a consequence, plants have developed active, inducible, and tightly regulated immune systems that mediate interactions with microbes. The outcome of these complex interactions is a determining factor for plant survival and fitness [1]. Moreover, plants have developed a priming system, whereby a previous localized attack can be memorized, at distal sites, and result in enhanced resistance [2]. For the purpose of this review, we briefly introduce the general concepts of innate immunity, defense signaling, and epigenetic memory before reviewing the crossover of these topics.

\section{Plant Innate Immunity}

Several models have been proposed for describing plant immune responses [3-5]. However, all models concur that the innate immune system is based on the perception of pathogen- and/or microbial-associated molecular patterns (PAMPs/MAMPs; see Glossary) and/or microbial effector proteins [6-8]. It is generally accepted that the first line of defense reactions in plants is triggered by the recognition of PAMPs by cell surface pattern recognition receptors (PRRs). Several PAMPs have been identified, the most commonly studied being flg22 (an evolutionarily conserved 22-residue peptide of bacterial flagellin) [9], the elf18 epitope of the bacterial elongation factor-Tu (EF-Tu), and chitin (a component of fungal cell walls) [10,11]. PAMP or damage-associated molecular patterns (DAMP) detection by PRRs triggers a complex network of intracellular signaling cascades, which lead to a series of defense responses known as PAMP-triggered immunity(PTI; Figure 1). PTI involves distinct well-characterized physiological phenomena, such as stomata closure to limit pathogen penetration, reactive oxygen species (ROS) and nitric oxide (NO) production, the limitation of nutritional transfer from the cytosol to the apoplast, callose deposition, and the biosynthesis of antimicrobial metabolites and defense hormones [9,12] (Figure 1). Major plant hormones that regulate defense responses include salicylic acid (SA), jasmonic acid (JA), and ethylene (ET). SA has a key role in defense against biotrophic pathogens, whereas JA and ET are critical to defense against necrotrophic

\section{Highlights}

To defend against microbial pathogens, plants have developed a sophisticated immune system. Upon recognition of external microbe-associated molecular patterns (MAMPs) or internal microbial effectors that are delivered into the host cells, signal transduction events are triggered, leading to rapid defense responses that include massive transcriptional reprogramming. These events are followed by the systemic activation of defense programs at distant, nonchallenged sites, a process termed 'systemic acquired resistance' (SAR) and linked to priming, whereby plants memorize previous attacks and can respond more robustly to subsequent pathogen challenges.

Recent evidence demonstrates that plant defense gene expression also involves epigenetic mechanisms, such as DNA methylation and histone modifications, that are closely linked to the dynamical chromatin states.

${ }^{1}$ Desert Agriculture Initiative, 4700 King Abdullah University of Science and Technology, Thuwal 23955-6900, Saudi Arabia

${ }^{2}$ Institut des Sciences des Plantes de Paris Saclay, IPS2, Bâtiment 630, Plateau du Moulon, Rue Noetzlin CS 80004, 91192 Gif-sur-Yvette, France ${ }^{3}$ Department of Biology, Science and Arts College, Rabigh Campus, King Abdulaziz University, Jeddah 21589, Saudi Arabia

${ }^{4}$ These authors contributed equally

${ }^{*}$ Correspondence:

moussa.benhamed@ips2.universiteparis-saclay.fr (M. Benhamed) and heribert.hirt@kaust.edu.sa (H. Hirt). 


\section{Trends in Plant Science}

pathogens and herbivores. Although the SA and JA/ET pathways often function antagonistically, analysis of a SA-, JA-, and ET-defective mutant suggests a synergistic mechanism whereby all three hormones contribute to defense $[13,14]$.

Due to the long coevolutionary history of plant-pathogen relationships, specialized pathogens have evolved means to suppress PTI. In this way, pathogenic microorganisms with different lifestyles and evolutionary origins have generated a diverse repertoire of effector proteins that are translocated into the plant cell, where they can affect protein or gene activity and promote disease, a phenomenon known as effector-triggered susceptibility (ETS) [3]. However, plants can use transmembrane or intracellular receptors, so-called 'resistance $(\mathrm{R})$ proteins', for the detection of these effectors. Effector recognition leads to effector-triggered immunity $(\mathrm{ETI})$, a process that overlaps with PTI to a great extent (Figure 1). It is generally accepted that, in contrast to PTI, ETI induces stronger and long-lasting responses, thereby inducing programmed cell death, a process known as the hypersensitive response $(\mathrm{HR})$, leading to pathogen resistance $[4,12]$ (Figure 1). The overlap between the physiological responses involved in PTI and ETI are due, at least partially, to convergence between the signaling induced by both pathways: several proteins act downstream of these signaling cascades, such as the transcription factors SARD1 and CBP60g [4]. This phenomenon leads to the activation of common immunity-related genes, some of which are used as defense markers, including PATHOGEN RELATED GENE-1 and 2 (PR1 and PR2), and FLG22-INDUCED RECEPTOR KINASE-1 (FRK1) [15,16].

There is growing evidence indicating that transcriptional reprogramming downstream of the signaling cascades involved in PTI and ETI requires the action of a highly diverse group of molecules in the plant nucleus. This process includes transcription factors, long noncoding RNAs (IncRNAs), small RNAs (sRNAs), histone modifiers, chromatin remodelers, and transcriptional regulatory complexes, among others [17-23]. Currently, little is known about how these elements interact to influence transcription or the molecular mechanisms by which they regulate immune responses.

\section{Defense and Epigenetics}

One of the first pieces of evidence for epigenomic regulation of plant immunity appeared with the description of the control of viral virulence through RNA silencing. In response to viral infections, plants recognize viral double-strand RNA molecules (dsRNAs), inducing their degradation into virus-derived small interference RNAs (vsiRNAs) by DICER-LIKE (DCL)-2 and DCL4 (proteins required for post-transcriptional gene silencing, PTGS; Figure 2A) [2426]. Generally, transcript decay leads to a significant decrease in viral protein production, together with increased resistance $[27,28]$. However, another mechanism, transcriptional gene silencing (TGS), adds an extra, and more stable, layer of defense against DNA viruses [29]. The process by which TGS occurs is known as RNA-directed DNA-methylation (RdDM) and involves the deposition of repressive methyl groups in cytosines of target sequences. The canonical RdDM involves a 24-nucleotide (nt) siRNA resulting from the activity of DCL3, which is loaded into the AGO4 protein and directs the RdDM machinery towards the target loci [30,31] Often, the deposition of DNA methylation is correlated with the addition of other repressive marks, such as the monomethylation of lysine 9 of Histone $3(\mathrm{H} 3 \mathrm{~K} 9 \mathrm{me})$, which reinforces the repressive effect of methylcytosines [32] (Figure 2A). Such a correlation can be seen in Arabidopsis DNA methylation mutants, such as $d d m 1$ and met1, where histone methylation and virus resistance levels are decreased [33,34].

PTGS and TGS are critical, but not limited, to virus defense; in fact, plants use such mechanisms for the regulation of their own gene expression. For instance, the RdDM pathway is

\section{Glossary}

Effector-triggered immunity (ETI): defense responses that are triggered by the direct or indirect recognition of a effector by a resistance protein. Microbe-associated molecular patterns (MAMPs): molecules that are typical for a microbe and foreign to a plant.

PAMP-triggered immunity (PTI): defense responses that are triggered by PAMP-triggered PRR.

Pattern recognition receptor (PPR): a sensor that detects the presence of a MAMP.

Systemic acquired resistance (SAR): defense responses that are triggered in the entire plant and over a longer period of time.

Transcriptional gene silencing (TGS): inhibition of expression of foreign or transgenes. 


\section{Trends in Plant Science}

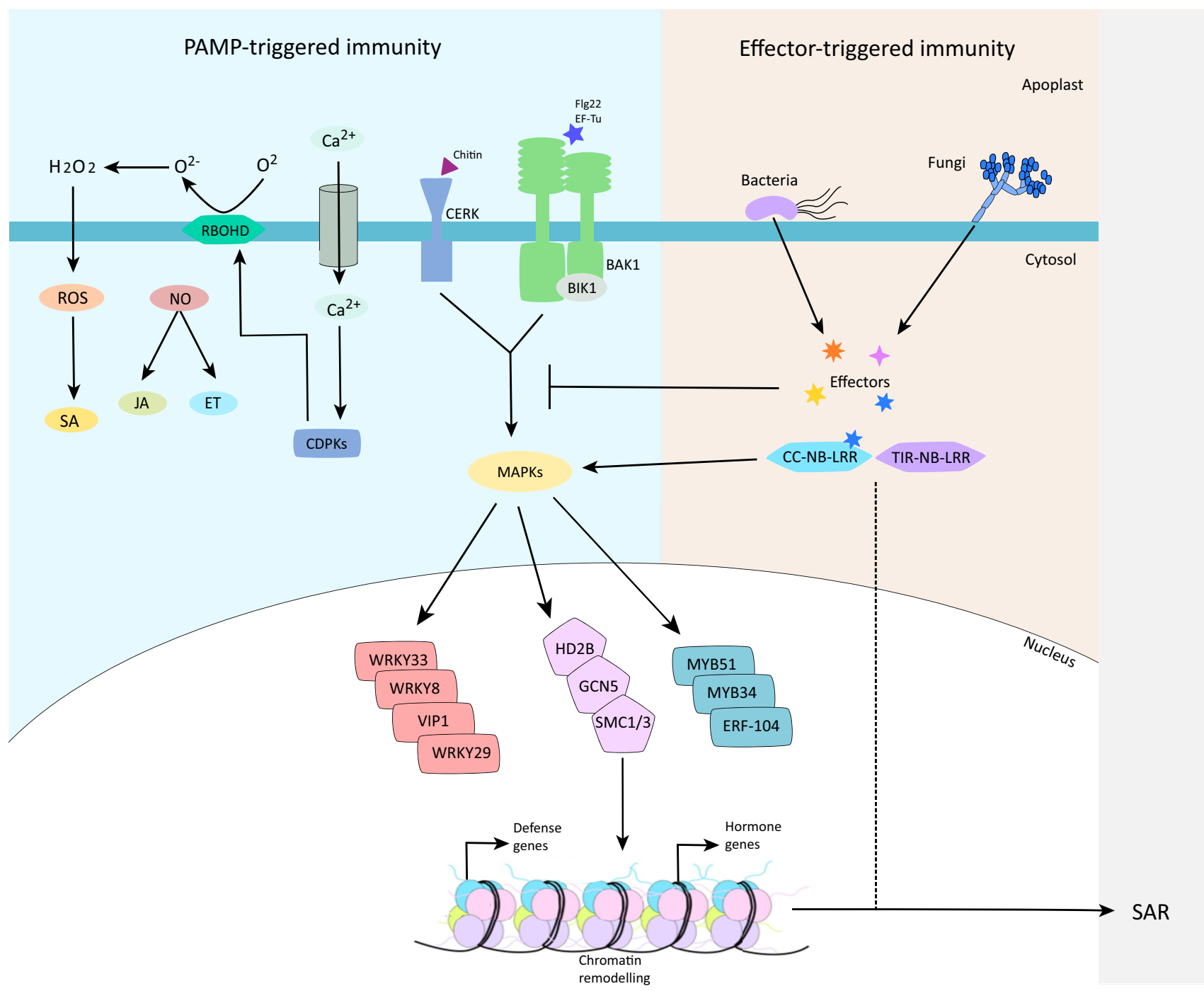

Trends in Plant Science

Figure 1. Defense Signaling in Plants. Perception of microbe-associated molecular patterns [MAMPS; flagellin, elongation factor-Tu (EF-Tu), and chitin] by the cognate pattern recognition receptors (PRRs; FLS2, EFR, and CERK) activates several signaling events such as reactive oxygen species (ROS) production, nitric oxide (NO), $\mathrm{Ca}^{2+}$ flux, and induction of different protein kinases, namely MAP kinases (MAPKs) and CDPKs. Pathogens deliver effector molecules into the plant to suppress these early signaling events. However, certain plant varieties can recognize effectors with the help of R proteins (CC-NB-LRR and TIR-NB-LRR) to induce a hypersensitive response (HR) and systemic acquired resistance (SAR). Targets that are phosphorylated include the transcription factors WRKY33, WRKY8, WRKY29, VIP1, MYB51, MYB34, and ERF104, and the chromatin remodeling factors HD2B, GCN5, and SMC1/3. This activation has a role in not only transcriptional reprogramming and induction of early defense-related genes, but also in limiting pathogen infection and priming plants against future attacks. Endogenous phytohormones, such as salicylic acid (SA), jasmonic acid (JA), and ethylene, are also induced and contribute to plant immunity.

essential for the repression of transposable elements (TEs), and its reactivation can have detrimental effects on genome stability and integrity $[35,36]$. It has been proposed that, in the absence of an adaptive immune system, plants have developed the most complex RNA-based regulation of gene expression within the eukaryotes (four different pathways of RNA silencing) to defend themselves against foreign nucleic acids [26,37]. These pathways have reached such degrees of sophistication in angiosperms that they involve some unique proteins and RNA 


\section{Trends in Plant Science}

(A)

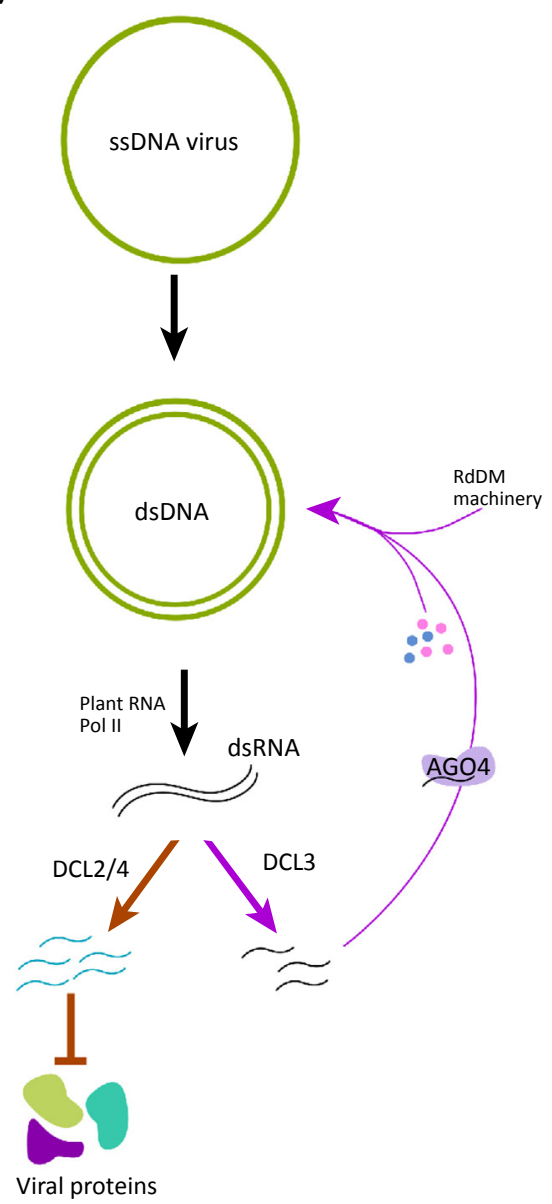

(B)

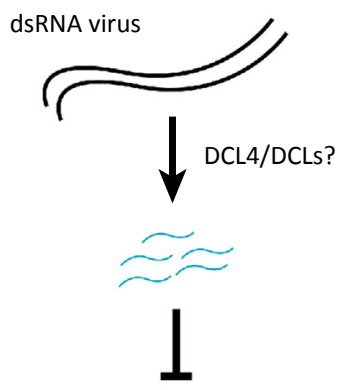

Viral replication

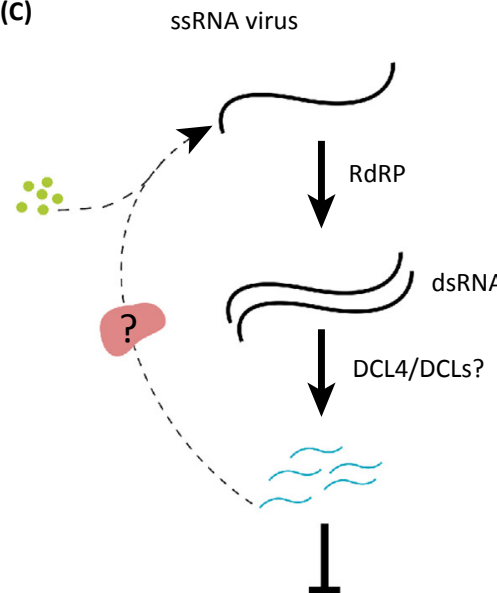

Viral replication

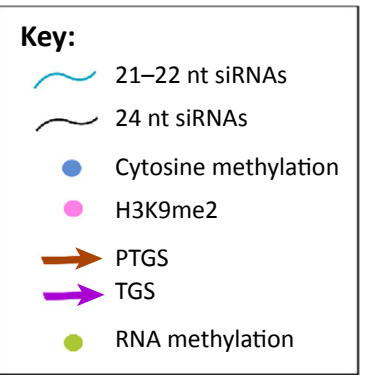

Trends in Plant Science

Figure 2. Plant Defense against Viral Pathogens Requires the Activity of DICER-LIKE (DCL) Proteins. (A) Plants can make use of transcriptional gene silencing (TGS), or post-transcriptional gene silencing (PTGS) pathways to control the replication of DNA viruses. Single-strand (ss)-DNA viruses, such as geminiviruses, use the host replication machinery to produce an intermediary double-strand (ds)-DNA molecule. Transcription from these dsDNAs by plant Pol-II leads to the formation of dsRNA molecules that can be recognized by the host immune system. Such recognition triggers the recruitment of DCL2 and DCL4, proteins that dice these transcripts into 24-nucleotide (nt) virus siRNAs (vsiRNAs), inhibiting their translation and the consequent formation of viral particles (via PTGS). Alternatively, these dsRNAs can be processed by DCL3 into 21-22-nt vsiRNAs, which are loaded onto AGO4 and guide the RNA-directed DNA methylation (RdDM) machinery towards the viral genome, where DNA and histone methylation marks are deposited, inhibiting its transcription. (B) dsRNA viral genomes can be directly diced by DCL proteins, including DCL4, inhibiting viral replication. (C) The genomes of sSRNA viruses are turned into dsRNA molecules via the activity of an RNA-dependent RNA polymerase. The resulting dsRNA molecules can be further processed by DCLs into vsiRNAs. In plants, RNA methylation was recently reported as a mechanism of viral immunity against members of the Bromoviridae; however, the proteins or complexes that perform such methylation are unknown, as well as the mechanism that guides them towards the viral genome. It seems reasonable to hypothesize that the vsiRNAs resulting from the viral genome degradation are implicated in the latter process.

polymerases essential for TGS (e.g., NERD, Pol-IV, Pol-V, RDM1, DMS3, and SHH1), even though this process is widely distributed among eukaryotes [38,39].

Equally relevant is the fact that DNA viruses are uncommon among plants, with single-stranded RNA viruses (ssRNA) being the most widespread of plant viruses. The immune response induced by both ssRNA and dsRNA viruses, occurring in many cases as a consequence of the recognition of virus-associated molecular patterns (VAMPs), triggers the degradation of their genomes and the consequent formation of vsiRNAs. However, dsRNAs can be directly 
targeted by DCLs (Figure 2B), while ssRNA genomes require the action of an RNA-dependent RNA polymerase (RDRP) to produce dsRNAs that can be processed by these proteins [40]. Recently, it was shown that RNA methylation, specifically N6-methyladenosine $\left(\mathrm{m}^{6} \mathrm{~A}\right)$, occurs in the genome of Bromovirus infecting Arabidopsis. Furthermore, the deposition of this mark on the viral genome was correlated with the control of viral infection in this plant [41] (Figure 2C). A similar phenomenon was previously observed in animal cells, where $\mathrm{m}^{6} \mathrm{~A}$ deposition on the genomes of different flaviviruses was linked to a decrease in viral replication [42,43]. Such results suggest the existence of a new epigenetic level of regulation of innate immunity, common between these distant phyla; however, the study of RNA post-transcriptional modifications is a particularly new field in plant biology, and only a few of the protein complexes involved in this process have been determined [44], resulting in several challenges and opportunities for understanding new mechanisms regulating immunity in these organisms.

By contrast, the role of DNA methylation in development and immunity has been exhaustively characterized in plants and other organisms $[45,46]$. As reviewed by Espinas and collaborators, the methylation of DNA has been linked with the regulation of PTI in various studies: in general, the mutation or downregulation of DNA methylation pathways, both in Arabidopsis (met1-3 and the triple $d r m$ 1-2 drm2-2 cmt3-11 mutants) and rice, leads to increased pathogen resistance levels and increased expression of defense-related genes [47-49]. There is compelling evidence indicating that DNA methylation negatively regulates defense in plants [48-50]; however, a recent study in Arabidopsis showed that mutants impaired in the deposition of these marks (ddm1, nrpe1, drd1, and cmt3) displayed increased resistance to the biotrophic pathogen Hyaloperonospora arabidopsidis, while presenting a higher susceptibility to the necrotrophic fungus Plectosphaerella cucumerina. The hypermethylated ros 1 mutant presented an inverse phenotype to the studied hypomethylated mutants, suggesting that DNA methylation negatively regulates defense responses against biotrophic pathogens, while positively regulating immunity against necrotrophs [51].

As an additional level of complexity of the molecular networks involved in the regulation of immunity, plants count on a considerable number of ncRNAs that have been associated with the transcriptional reprogramming that occurs during stress responses. Several sRNAs and IncRNAs have been reported to participate in TGS and PTGS, regulating several crucial aspects of defense, as well as the hormone balance [18,19]. miR393 was the first sRNA to be identified as being involved in PTI, where it downregulates auxin signaling by negatively regulating receptors for this hormone [52]. This miRNA has also been related to the regulation of levels of camalexin and glucosinolates, which are secondary metabolites with significant roles in Arabidopsis defense [53]. Several other miRNAs have been associated with immunity in various plant species, where their roles range from regulating auxin levels, callose deposition, and $R$ gene expression to ROS production, among others [19,54].

Technical advances in sequencing methods have permitted the identification of various IncRNAs that are differentially induced in plants in response to specific stresses [55-59]. These molecules, of more than $200 \mathrm{nt}$, can affect several cellular processes, including TGS, miRNA and protein hijack, transcription regulation of protein-coding genes in cis and trans, and the modification of chromatin at diverse levels $[60,61]$. However, in contrast to animals, only a few IncRNAs have been functionally characterized in plants. An initial study identified various Arabidopsis polyadenylated IncRNAs induced by elf18 treatment [62]. More recently, the role of one of these molecules was characterized in the regulation of immune responses: ELENA1 expression was shown to be induced by flg22 and elf18, and plants deficient in their perception did not display any increased levels of this IncRNA in response to these PAMPs, showing that 


\section{Trends in Plant Science}

ELENA1 transcription occurs as the result of a PTI-induced signaling cascade. Furthermore, in the same study, ELENA1-knockdown and -overexpressing lines were found to display increased susceptibility and resistance to Pseudomonas syringae pv. tomato DC3000 (Pst DC3000), respectively. Lines overexpressing this locus presented higher levels of transcription in PR genes, such as PR1,PR2, and other SA-induced genes, a phenomenon that appears to be due to the interaction of ELENA1 and the MED19A subunit of the Mediator complex, which mediates the interaction between Pol-II and transcription factors. Even though there is strong evidence indicating that ELENA1 regulates the expression of some PTI-induced genes in trans, the mechanisms by which this IncRNA specifically targets these loci remain unknown $[20,63]$. By contrast, through a strand-specific RNA sequencing approach, Zhu and collaborators detected several IncRNAs induced in Arabidopsis in response to infection by the fungal pathogen Fusarium oxysporum. In this study, the authors detected various natural antisense transcripts (NATs) and 20 Fusarium-responsive long noncoding transcriptionally active regions (IncTARs), of which ten were characterized through knockdowns or T-DNA insertions. Knockout mutants for some of these IncTARs presented higher susceptibility to this fungus, indicating their role in the regulation of defense. Additionally, promoter analyses suggested that some of these IncTARs are direct targets of transcription factors that respond to pathogen attack; however, and similar to ELENA1, the exact molecular mechanisms by which they act remain to be elucidated $[57,58]$.

Together with DNA methylation, IncRNAs, and sRNAs, histone modifications have a significant role in the regulation of transcriptional regulation in eukaryotes [64,65]. As previously discussed, several chromatin and histone modifiers and remodelers have also been associated with plant defense $[23,66]$. In fact, immunity appears to be coordinated, at least partially, by numerous epigenomic processes and histone post-translational modifications with both antagonistic and synergistic activities over transcription and defense [67]. This is how various histone deacetylases and acetyltransferases (HDACs and HATs, respectively), methylases, demethylases, ubiquitinases, and chromatin remodelers have been found to act on different aspects of immunity, and some have been described as positive and negative regulators of either specific aspects of, or overall immunity [66].

It is thought that histone acetylation, a mark occurring preferentially in lysines, perturbs the interactions between nucleosomes, leading to more accessible chromatin and increased transcription [68]. So far, two Arabidopsis HATs and four HDACs have been reported to be directly involved in the regulation of defense [23,69-77]. The elongator complex, which facilitates transcription by modifying chromatin in a co-transcriptional manner, has been proposed to be a positive regulator of immunity. ELP3, its catalytic subunit and a protein with acetyltransferase activity, was found to be required for basal immunity and ETI, since its mutants showed decreased SA levels, delayed defense gene expression, and higher susceptibility to $P$. syringae pv. maculicola (Psm) [77]. Concomitantly, another elongator subunit, ELP2, also proved to be necessary for fast transcriptional reprogramming after pathogen attack, and the complete induction of the JA/ET pathway in response to necrotrophic fungi: lack of this protein led to enhanced susceptibility to Botrytis cinerea and lack of induction of JAdefense marker genes, including PDF1.2, WRKY33, and ORA59 [78,79]. Among the Arabidopsis HDACs, HDA19 and HDA6 were proposed to result in redundancy in the regulation of immunity, since both proteins were reported as positive regulators of JA-mediated defense, while acting as repressors of SA pathways against biotrophs [23,69-73,80]. Similarly, the SRT2 deacetylase was proposed to negatively regulate SA responses, and its mutant was found to present increased resistance to PstDC3000 [75]. A recent integrative study reported that pathogen-triggered SA signaling induced the biosynthesis of $\mathrm{NO}$, a molecule that physically 
inhibits several HDACs, including HDA19. Consequently, such inhibition leads to elevated acetylation levels in several SA defense genes and their transcription [81], a phenomenon that correlates with the described negative role of this HDAC over SA-mediated defense. It has been proposed that histone acetylation positively regulates SA-mediated defenses, since most of the plant HDAC mutants studied so far have been reported to be more resistant to at least one biotrophic or hemibiotrophic pathogen, including the rice deacetylase HDT701 $[47,82]$. However, a recent study reported that the HD2B deacetylase mutant presented increased susceptibility to PstDC3000 [74], indicating that HD2B-mediated deacetylation is important for the establishment of appropriate basal defense. Furthermore, the study established a direct connection between the signaling of a pathogen to transcriptional reprogramming through histone modifications. HD2B was identified as a direct MPK3 target that is phosphorylated upon MAMP-triggered activation of the MAPK cascade. Once phosphorylated, HD2B is mobilized from the nucleolus to the nucleoplasm to perform the deacetylation and fine-tuning of defense responses [74].

Several histone methyltransferases and demethylases have been described as immunity regulators. In fact, most of the mutants of both types of enzyme display compromised resistance to pathogens, suggesting an important role of the regulation of histone methylation in defense. However, it would be difficult to determine whether these marks regulate immunity positively or negatively, as has been previously proposed for histone acetylation. This phenomenon may be due to the fact that the impact of different histone methylations over transcription is more varied than that of acetylation, which is mainly an activating mark. However, similar roles in the regulation of immunity may occur in related proteins. For instance, some proteins belonging to the Jumonji-C demethylase family contribute to immunity by repressing the expression of negative regulators of defense: Arabidopsis JMJ27 represses WRKY25 and WRKY33 by removing H3K9me1/2, while rice JMJ704 is necessary for the repression of OsWRKY66, NRR, and Os-11N3 via H3K4me2/3 demethylation [83,84]. Among the methyltransferases that have been reported to have a role in immunity are ATX1, SDG25, and SDG8, mutants of which are defective in basal defense, necrotroph resistance, and SAR, respectively [85-88].

In recent years, the importance of histone ubiquitination and chromatin remodeling in the regulation of defense has become evident; however, these phenomena have been less explored compared with the previously discussed histone marks. Specifically, H2B monoubiquitination (H2Bub1) was found to be necessary for the expression of the R genes RPP4 and SNC1 in Arabidopsis, as well as for effective microtubule dynamics in response to the fungus Verticillium dahliae $[89,90]$. Furthermore, mutation of the HUB1 locus, encoding one of the two Arabidopsis H2Bub1 ligases, led to increased sensitivity to necrotrophic fungi, confirming the function of these marks in plant defense [91]. Similar to HUB1, the Arabidopsis SWI/SNF class chromatin remodeling ATPase SPLAYED (SYD) was described to be crucial for defense against B. cinerea but not PstDC3000, mediating the expression of JA/ET-responsive genes and being a positive regulator of defense against necrotrophs [92]. By contrast, the SWR1 chromatinremodeling complex, which performs the substitution of canonical $\mathrm{H} 2 \mathrm{~A}$ histones by the histone variant H2A.Z, was described as a particular regulator of immunity, since mutation of its subunits led to completely different phenotypes: lack of pie1 and swc6 induced a compromised basal resistance and ETI, while arp6 loss of function enhanced it [93,94]. This last phenomenon represents just a hint of the high levels of complexity that chromatin organization has reached in the plant kingdom, for the regulation of immunity and, probably, every other physiological process. 
Similar to RdDM, histone modifiers and chromatin remodelers have, in most cases, a genomewide function, and are involved in the transcriptional reprogramming of highly complex processes, such as flowering and immunity. Thus, it could be argued that studying mutants of these proteins for their resistance levels to different pathogens, and from this classifying them as positive or negative regulators of defense, is both simplistic and dualistic. Alternatively, it could be proposed that, because immunity involves enormous transcriptional reprogramming, it is a sensitive system that depends on hundreds of proteins and ncRNAs regulating these transcriptional changes. The elimination of one of these elements could lead to relatively small effects that could be interpreted as immunity phenotypes, even when this element is not directly involved in defense. Nevertheless, because the study of the epigenetic regulation of immunity in plants is a relatively new field, these characterizations, as simplistic as they may appear, represent a first approach towards the understanding of the mechanisms involved.

\section{Innate Immunity and Epigenetic Memory}

The term 'priming' refers to the process by which an acute environmental stress modifies the plant response to a future challenge. This phenomenon was first described in the study of biotic interactions, where initial exposure to a pathogenic microorganism led to a faster and stronger defense response after subsequent attacks, without affecting any DNA sequences. However, this process is also triggered by abiotic stresses, including cold, heat, drought, and salinity $[95,96]$. Priming involves a subsequent stress memory that is partially mediated by the accumulation of inactive MAP kinases, changes in DNA methylation, and histone modifications: in fact, MPK3 and MPK6 are necessary for full priming of defense genes [97]. There is also evidence that priming can affect somatic cells and be temporal (epigenetic sensu lato) or, in a few cases, such as chromatin in gametes, can have a transgenerational effect (epigenetic sensu stricto). However, the latter has only been observed to be inherited through a limited number of generations in a few studies [96,98-101].

SAR was shown to induce changes in histone modification levels, especially H3K4me2 and H3K4me3, at different loci of pathogen-inoculated and distal tissues [88,102]. Both histone marks are generally associated with transcriptional activation and it is thought that they poise specific loci for gene expression [102]. Interestingly, one type of stress can also prime plants for another stress (cross-priming): for instance, Arabidopsis plants exposed to sublethal levels of salt, cold, and heat were found to be more resistant to infection by bacterial pathogens, and to present modified histone modification levels and a more open chromatin state in various PTI marker genes (including WRKY53, FRK1, and NHL10). Furthermore, the histone acetyltransferase 1 (hac1) mutant was found to be impaired in the establishment of this cross-stress priming, presenting an increased susceptibility to infection compared with wild-type plants after abiotic stress challenging [76]. However, unchallenged mutants did not display any compromised pathogen resistance, indicating that the action of this histone acetyltransferase is indispensable for priming of PTI genes but not for PTI per se [76]. Generally, these studies use flower dipping or bacterial spraying as inoculation methods, since pathogen infiltration does not induce priming, indicating that stomatal regulation is necessary for the induction of this process. However, such phenomenon imposes a limitation, since the dipping and spraying methods are unlikely to ensure homogenous infection levels throughout the plant tissue. Equally, it is almost impossible to select only infected tissue for performing transcriptomic or proteomic studies, which could lead to artifacts and discrepancies among studies of these processes.

Given their high energetic cost, defense responses are repressed when unnecessary and plants use a sophisticated system to suppress immune processes and priming. An Arabidopsis 
mutant deficient in the FAS2 subunit of the histone chaperone CAF1 shows constitutive priming of defense genes and is characterized by low nucleosome occupancy and increased H3K4me3 levels in defense genes. The importance of CAF1-mediated repression of priming can be evidenced in the developmental defects presented by this mutant, which is considerably smaller and less vigorous and fertile than the wild-type, probably due to its increased SA responsiveness, which in many cases can be associated with the downregulation of auxin signaling [103]. Even when it is less costly for a plant to maintain a primed state instead of a complete defense response, it could be advantageous to reset such a state after several days of 'being prepared' for a new stress that may or may not arrive: a constitutive priming could eventually represent a fitness cost instead of a benefit $[96,104]$.

The study of transgenerational memory (epigenetics sensu stricto) of stress is a relatively new area of research that presents several difficulties and challenges. Nevertheless, there is some evidence for this poorly understood phenomenon. The heritability of priming states has been evidenced with different stresses; however, only a few studies have demonstrated the vertical transmission of defense-induced epialleles. Two studies found that PstDC3000-induced epigenomic changes can be inherited by the first and second stress-free generations, with several SA-responsive promoters hyperacetylated in $\mathrm{H} 3 \mathrm{~K} 9$ and showing higher pathogen and SA responsiveness (Figure 3) [98,99]. A similar phenomenon was observed with herbivoreinduced priming (which triggers the JA-defense pathway in a similar way to necrotrophs), a phenomenon where the progeny (F1 and F2) of caterpillar-attacked Arabidopsis and tomato plants displayed increased herbivore resistance in a JA-dependent fashion [100]. More recently, a study performed in common bean (Phaseolus vulgaris) found that the PTI marker pvPR1 gene can be primed after attack by $P$. syringae pv. phaseolicola, and the new epiallele transferred to its progeny. The authors proposed that this could be exploited by crop breeders,

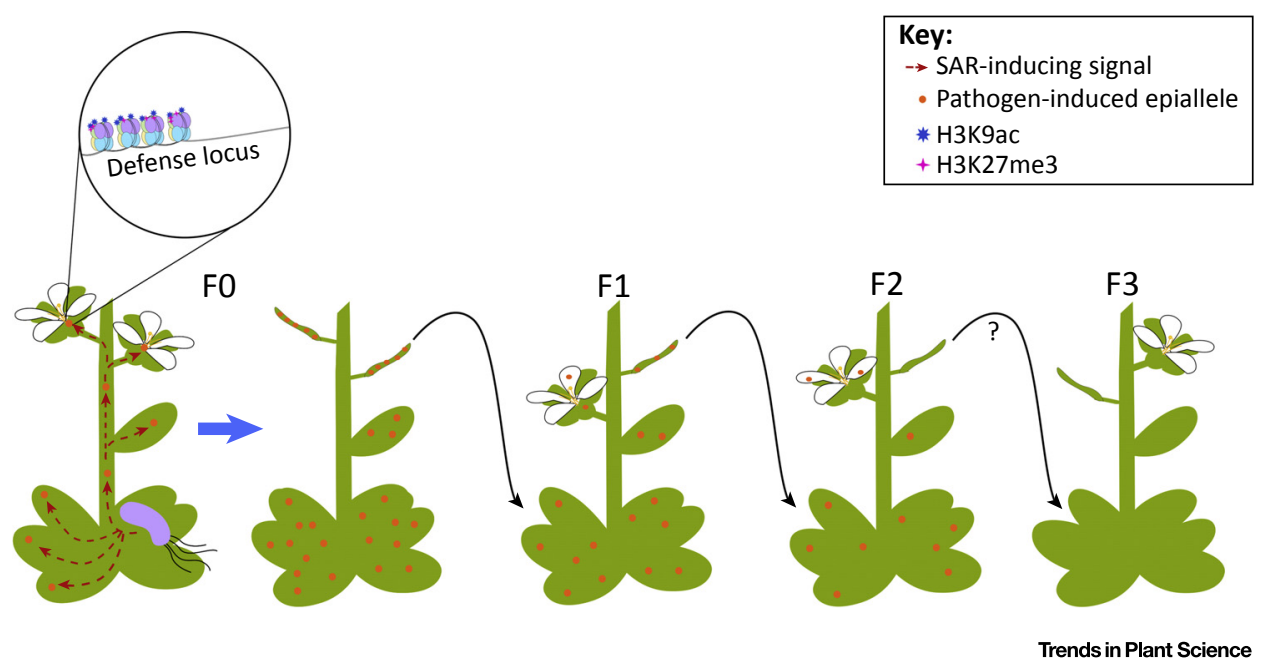

Figure 3. Transgenerational Transmission of Pathogen-Induced Priming. Even when there is limited evidence for the vertical transmission of pathogen-induced epialleles, this phenomenon has been reported by a growing number of studies, performed in diverse plants. It has been proposed that systemic acquired resistance (SAR), induced by pathogen detection, triggers a systemic priming of pathogen-responsive genes, which includes changes in histone methylation and acetylation levels in their promoter sequences. The newly generated epialleles, present in reproductive tissue, can be inherited by the progeny, where they display higher inducibility than the original epialleles upon pathogen attack, conferring increased resistance to disease. The extent to which such a pathogen-induced transgenerational effect is stable through generations remains to be determined, since it could represent a considerable energetic cost in the absence of pathogen attack. 


\section{Trends in Plant Science}

who could intentionally prime plants before crossing them and select for epialleles conferring increased disease tolerance [101]. Such a mechanism could offer interesting possibilities; however, so far, there are no reports of stable pathogen-induced priming persisting through more than two generations, which could impose a limitation to the fixing of desired epialleles in agronomic populations. We hypothesize that this long-term transgenerational instability of stress-induced priming results from the possible energetic or homeostatic disadvantages of maintaining this priming in the absence of stress: upon several stress-free generations, the selection force driving the maintenance of a primed state would disappear, leading to resetting of the epigenomic state of the former primed loci.

\section{Concluding Remarks}

There is still a considerable lack of studies establishing the mechanisms by which signaling is linked to the epigenetic memory of plant defense. It is evident that we still have a long way to go towards understanding the exact function of the epigenomic machinery in the regulation of defense and other biological processes. Although with current molecular technologies, we are able to generate correlations between a specific input (or stimulus), a genotype, and an output (phenotype), most cellular molecules, mechanisms, interactions, and steps that drive physiological responses are still to be fully understood. For instance, in animal cells, it is known that the activation of the MAPK transduction pathway can lead to phosphorylation of histone $\mathrm{H} 3$ $[105,106]$. However, no direct MAPK histone phosphorylation has yet been observed in plants. This could be because either we have not been able to detect these modifications due to technical challenges, or the innate immune systems of plants and animals have diverged in this aspect. However, the innate immune system of plants, invertebrates, and humans may harbor more similarities than hitherto assumed. In vertebrates, which use both an innate and adaptive immune system, the dogma that long-lasting immunity is only conveyed by $\mathrm{T}$ and $\mathrm{B}$ cells has been challenged by recent work [107], suggesting that immune memory is also provided by the innate immune system. These findings add relevance to more fully understanding how plants and invertebrates, which use only an innate immune system, can not only defend against an acute attack, but may also use previous information to respond more effectively to subsequent challenges. Therefore, understanding the epigenetic basis of innate immune memory, and the degree to which it may be transgenerational, could result in unexpected 'vaccination' strategies, both in humans and in agriculture (see also Outstanding Questions).

\section{References}

1. Brader, G. et al. (2014) Metabolic potential of endophytic bacteria. Curr. Opin. Biotechnol. 27, 30-37

2. Martinez-Medina, A. et al. (2016) Recognizing plant defense priming. Trends Plant Sci. 21, 818-822

3. Cui, H. et al. (2015) Effector-triggered immunity: from pathogen perception to robust defense. Annu. Rev. Plant Biol. 66, 487511

4. Peng, Y. et al. (2018) Convergent and divergent signaling in PAMP-triggered immunity and effector-triggered immunity. Mol, Plant Microbe Interact. 31, 403-409

5. Van Der Biezen, E. and Jones, J.D.G. (1998) Plant diseaseresistance proteins and the gene-for-gene concept. Trends Biochem. Sci. 23, 454-456

6. Nürnberger, T. and Brunner, F. (2002) Innate immunity in plants and animals: emerging parallels between the recognition of general elicitors and pathogen-associated molecular patterns. Curr. Opin. Plant Biol. 5, 318-324

7. Boller, T. and Felix, G. (2009) A renaissance of elicitors: perception of microbe-associated molecular patterns and danger signals by pattern-recognition receptors. Annu. Rev. Plant Biol. 60, 379-406
8. Bent, A.F. and Mackey, D. (2007) Elicitors, effectors, and R genes: the new paradigm and a lifetime supply of questions. Annu. Rev. Phytopathol. 45, 399-436

9. Bigeard, J. et al. (2015) Signaling mechanisms in pattern-triggered immunity (PTI). Mol. Plant 8, 521-539 factor Tu elicits innate immunity in Arabidopsis plants. Plant Cell Online 16, 3496-3507

11. Hayafune, M. et al. (2014) Chitin-induced activation of immune signaling by the rice receptor CEBiP relies on a unique sandwich-type dimerization. Proc. Natl. Acad. Sci. 111, E404E413

12. Zhang, J. and Zhou, J.M. (2010) Plant immunity triggered by microbial molecular signatures. Mol. Plant 3, 783-793

13. Meng, X. and Zhang, S. (2013) MAPK cascades in plant disease resistance signaling. Annu. Rev. Phytopathol. 51, 245-266

14. Pieterse, C.M.J. et al. (2009) Networking by small-molecule hormones in plant immunity. Nat. Chem. Biol. 5, 308-316

15. Yeh, Y.-H. et al. (2015) Enhanced Arabidopsis pattern-triggered immunity by overexpression of cysteine-rich receptor-like kinases. Front. Plant Sci. 6, 322
10. Kunze, G. et al. (2004) The $\mathrm{N}$ terminus of bacterial elongation

\section{Outstanding Questions}

Can MAMPs and microbial effectors both induce long-term innate memory?

If MAMPs and effectors induce innate memory, do they do this by the same mechanisms?

Can MAMP- and effector-triggered immune memory be combined to obtain enhanced long-term responses?

Which epigenetic factors are targeted by MAMP- and which by effector-triggered signaling pathways?

Do biotic and abiotic stress memories differ with respect to their epigenetic pathways and mechanisms? 


\section{Trends in Plant Science}

16. De-La-Peña, C. et al. (2012) Regulation of disease-responsive genes mediated by epigenetic factors: interaction of Arabidopsis-Pseudomonas. Mol. Plant Pathol. 13, 388-398

17. Li, B. et al. (2016) Transcriptional regulation of pattern-triggered immunity in plants. Cell Host Microbe 19, 641-650

18. Huang, J. et al. (2016) Diverse functions of small RNAs in different plant-pathogen communications. Front. Microbiol. 7 , $1-17$

19. Huang, J. et al. (2016) The function of small RNAs in plant biotic stress response. J. Integr. Plant Biol. 58, 312-327

20. Mach, J. (2017) The long-noncoding RNA ELENA1 functions in plant immunity. Plant Cell 29, 916-916

21. Ramirez-Prado, J.S. et al. (2018) Modify the histone to win the battle: chromatin dynamics in plant-pathogen interactions. Front. Plant Sci. 9, 355

22. Nejat, N. and Mantri, N. (2017) Emerging roles of long noncoding RNAs in plant response to biotic and abiotic stresses. Crit. Rev. Biotechnol. 8551, 1-13

23. Ding, B. and Wang, G.-L. (2015) Chromatin versus pathogens: the function of epigenetics in plant immunity. Front. Plant Sci. 6, 675

24. Wieczorek, P. and Obrępalska-Stęplowska, A. (2015) Suppress to survive-implication of plant viruses in PTGS. Plant Mol. Biol. Rep. 33, 335-346

25. Vaucheret, H. et al. (2001) Post-transcriptional gene silencing in plants by RNA. J. Cell Sci. 114, 3083-3091

26. Agius, C. et al. (2012) RNA silencing and antiviral defense in plants. Methods Mol. Biol. 894, 17-38

27. Gambino, G. and Pantaleo, V. et al. (2017) Epigenetics in plantpathogen interactions. In Plant Epigenetics (Rajewsky, N., ed.), pp. 385-404, Springer Naure

28. Unver, T. and Budak, H. (2009) Virus-induced gene silencing, a post transcriptional gene silencing method. Int. J. Plant Genomics 2009, 198680

29. Pumplin, N. and Voinnet, O. (2013) RNA silencing suppression by plant pathogens: defence, counter-defence and countercounter-defence. Nat. Rev. Microbiol. 11, 745-760

30. Rajeev Kumar, S. et al. (2017) Small RNAs: master regulators of epigenetic silencing in plants. In Plant Epigenetics (Rajewsky, N., ed.), pp. 89-106, Springer

31. Holoch, D. and Moazed, D. (2015) RNA-mediated epigenetic regulation of gene expression. Nat. Rev. Genet. 16, 71-84

32. Du, J. et al. (2015) DNA methylation pathways and their crosstalk with histone methylation. Nat. Rev. Mol. Cell Biol. 16, 519532

33. Raja, P. et al. (2008) Viral genome methylation as an epigenetic defense against geminiviruses. J. Virol. 82, 8997-9007

34. Soppe, W.J.J. et al. (2002) DNA methylation controls histone $\mathrm{H}_{3}$ lysine 9 methylation and heterochromatin assembly in Arabidopsis. EMBO J. 21, 6549-6559

35. Vergara, Z. and Gutierrez, C. (2017) Emerging roles of chromatin in the maintenance of genome organization and function in plants. Genome Biol. 18, 1-12

36. McCue, A.D. et al. (2015) ARGONAUTE 6 bridges transposable element mRNA-derived siRNAs to the establishment of DNA methylation. EMBO J. 34, 20-35

37. Matzke, M.A. and Mosher, R.A. (2014) RNA-directed DNA methylation: an epigenetic pathway of increasing complexity. Nat. Rev. Genet. 15, 394-408

38. He, X. et al. (2009) A conserved transcriptional regulator is required for RNA-directed DNA methylation and plant development. Genes Dev. 23, 2717-2722

39. Ma, L. et al. (2015) Angiosperms are unique among land plant lineages in the occurrence of key genes in the RNA-directed DNA methylation (RdDM) Pathway. Genome Biol. Evol. 7, 2648-2662

40. Brodersen, P. and Voinnet, O. (2006) The diversity of RNA silencing pathways in plants. Trends Genet. 22, 268-280
41. Martínez-Pérez, M. et al. (2017) Arabidopsis $\mathrm{m}^{6} \mathrm{~A}$ demethylase activity modulates viral infection of a plant virus and the $\mathrm{m}^{6} \mathrm{~A}$ abundance in its genomic RNAs. Proc. Natl. Acad. Sci. 114 10755-10760

42. Brocard, M. et al. (2017) m6A RNA methylation, a new hallmark in virus-host interactions. J. Gen. Virol. 98, 2207-2214

43. Gokhale, N.S. et al. (2016) N6-methyladenosine in Flaviviridae viral RNA genomes regulates infection. Cell Host Microbe 20, 654-665

44. Ruzicka, K. et al. (2017) Identification of factors required for $m$ 6 A mRNA methylation in Arabidopsis reveals a role for the conserved E3 ubiquitin ligase HAKAl. New Phytol. 215, 157172

45. Zhang, M. et al. (2010) DNA cytosine methylation in plant development. J. Genet. Genomics 37, 1-12

46. Schuyler, R.P. et al. (2016) Distinct trends of DNA methylation patterning in the innate and adaptive immune systems. Cell Rep. 17, 2101-2111

47. Espinas, N.A. et al. (2016) Epigenetic control of defense signaling and priming in plants. Front. Plant Sci. 7, 1-7

48. Akimoto, K. et al. (2007) Epigenetic inheritance in rice plants. Ann. Bot. 100, 205-217

49. Dowen, R.H. et al. (2012) Widespread dynamic DNA methylation in response to biotic stress. Proc. Natl. Acad. Sci. 109, E2183-E2191

50. Yu, A. et al. (2013) Dynamics and biological relevance of DNA demethylation in Arabidopsis antibacterial defense. Proc. Nath. Acad. Sci. 110, 2389-2394

51. Lopez Sanchez, A. et al. (2016) The role of DNA (de) methylation in immune responsiveness of Arabidopsis. Plant J. 88, 361-374

52. Si-ammour, $\mathrm{A}$. et al. (2011) miR393 and secondary siRNAs regulate expression of the TIR1/AFB2 auxin receptor clade and auxin-related development of Arabidopsis leaves. Plant Physiol. 157, 683-691

53. Robert-Seilaniantz, A. et al. (2011) The microRNA miR393 re directs secondary metabolite biosynthesis away from camalexin and towards glucosinolates. Plant J. 67, 218-231

54. Huang, J. et al. (2016) Diverse functions of small RNAs in different plant-pathogen communications. Front. Microbiol. 7 , $1-17$

55. Shuai, P. et al. (2014) Genome-wide identification and functiona prediction of novel and drought-responsive lincRNAs in Populus trichocarpa. J. Exp. Bot. 65, 4975-4983

56. Shin, S.Y, and Shin, C. (2016) Regulatory non-coding RNAs in plants: potential gene resources for the improvement of agricul tural traits. Plant Biotechnol. Rep. 10, 35-47

57. Zhu, Q.H. et al. (2014) Long noncoding RNAs responsive to Fusarium oxysporum infection in Arabidopsis thaliana. New Phytol. 201, 574-58

58. Nejat, N. and Mantri, N. (2017) Emerging roles of long noncoding RNAs in plant response to biotic and abiotic stresses. Crit. Rev. Biotechnol. 8551, 1-13

59. Qi, X. et al. (2013) Genome-wide annotation of genes and noncoding RNAs of foxtail millet in response to simulated drought stress by deep sequencing. Plant Mol. Biol. 83, 459473

60. Ramirez-Prado, J.S. et al. (2017) Plant epigenetics: non-coding RNAs as emerging regulators. In Plant Epigenetics (Rajewsky, N., ed.), pp. 129-147, Springe

61. Ariel, F. et al. (2015) Battles and hijacks: noncoding transcription in plants. Trends Plant Sci. 20, 362-371

62. Liu, J. et al. (2012) Genome-wide analysis uncovers regulation of long intergenic noncoding RNAs in Arabidopsis. Plant Cell 24, 4333-4345

63. Seo, J.S. et al. (2017) ELF18-INDUCED LONG-NONCODING RNA associates with Mediator to enhance expression of innat immune response genes in Arabidopsis. Plant Cell 29, 1024 1038 
Trends in Plant Science

64. Smale, S.T. et al. (2014) Chromatin contributions to the regulation of innate immunity. Annu. Rev. Immunol. 32, 489-511

65. Obata, Y. et al. (2015) Epigenetic modifications of the immune system in health and disease. Immunol. Cell Biol. 93, 226-232

66. Ramirez-Prado, J.S. et al. (2018) Modify the histone to win the battle: chromatin dynamics in plant-pathogen interactions. Front. Plant Sci. 9, 355

67. Berr, A. et al. (2012) Chromatin modification and remodelling: a regulatory landscape for the control of Arabidopsis defence responses upon pathogen attack. Cell. Microbiol. 14, 829-839

68. Shahbazian, M.D. and Grunstein, M. (2007) Functions of sitespecific histone acetylation and deacetylation. Annu. Rev. Biochem. 76, 75-100

69. Zhou, C. et al. (2005) HISTONE DEACETYLASE19 is involved in jasmonic acid and ethylene signalling of pathogen response in Arabidopsis. Plant Cell 17, 1196-1204

70. Zhou, J. et al. (2010) Genome-wide profiling of histone H3 lysine 9 acetylation and dimethylation in arabidopsis reveals correlation between multiple histone marks and gene expression. Plant Mol. Biol. 72, 585-595

71. Choi, S.M. et al. (2012) HDA19 is required for the repression of salicylic acid biosynthesis and salicylic acid-mediated defense responses in Arabidopsis. Plant J. 71, 135-146

72. Wu, K. et al. (2008) HDA6 is required for jasmonate response, senescence and flowering in Arabidopsis. J. Exp. Bot. 59, 225234

73. Wang, Y. et al. (2017) HISTONE DEACETYLASE 6 represses pathogen defense responses in Arabidopsis thaliana. Plant Cell Environ. 40, 2972-2986

74. Latrasse, D. et al. (2017) MAPK-triggered epigenetic reprogramming by histone deacetylase in plant innate immunity. Genome Biol. 18, 131

75. Wang, C. et al. (2010) Arabidopsis putative deacetylase AtSRT2 regulates basal defense by suppressing PAD4, EDS5 and SID2 expression. Plant Cell Physiol. 51, 1291-1299

76. Singh, P. et al. (2014) Environmental history modulates Arabidopsis pattern-triggered immunity in a HISTONE ACETYLTRANSFERASE1-dependent manner. Plant Cell 26, 26762688

77. Defraia, C.T. et al. (2013) Elongator subunit 3 positively regulates plant immunity through its histone acetyltransferase and radical S-adenosylmethionine domains. BMC Plant Biol. 13, 102

78. Wang, C. et al. (2015) Arabidopsis Elongator subunit 2 positively contributes to resistance to the necrotrophic fungal pathogens Botrytis cinerea and Alternaria brassicicola. Plant J. 83, 10191033

79. Defraia, C.T. et al. (2010) Elongator subunit 2 is an accelerator of immune responses in Arabidopsis thaliana. Plant J. 64, 511-523

80. Kim, K.C. et al. (2008) Arabidopsis WRKY38 and WRKY62 transcription factors interact with histone deacetylase 19 in basal defense. Plant Cell 20, 1-2

81. Mengel, A. et al. (2017) Nitric oxide modulates histone acetylation at stress genes by inhibition of histone deacetylases. Plant Physiol. 173, 1434-1452

82. Ding, B. et al. (2012) HDT701, a histone H4 deacetylase, negatively regulates plant innate immunity by modulating histone $\mathrm{H} 4$ acetylation of defense-related genes in rice. Plant Cell 24, 37833794

83. Hou, Y. et al. (2015) JMJ704 positively regulates rice defense response against Xanthomonas oryzae pv. oryzae infection via reducing $\mathrm{H} 3 \mathrm{~K} 4 \mathrm{me} 2 / 3$ associated with negative disease resistance regulators. BMC Plant Biol. 15, 286

84. Dutta, A. et al. (2017) JMJ27, an Arabidopsis H3K9 histone demethylase, modulates defense against Pseudomonas syringae and flowering time. Plant J. 38, 42-49

85. Alvarez-Venegas, R. and Avramova, Z. (2005) Methylation patterns of histone H3 Lys 4, Lys 9 and Lys 27 in transcriptionally active and inactive Arabidopsis genes and in atx 1 mutants. Nucleic Acids Res. 33, 5199-5207
86. Alvarez-venegas, R. et al. (2007) Epigenetic control of a transcription factor at the cross section of two antagonistic pathways. Epigenetics 2, 106-117

87. Berr, A. et al. (2010) Arabidopsis histone methyltransferase SET DOMAIN GROUP8 mediates induction of the jasmonate/ethyene pathway genes in plant defense response to necrotrophic fungi. Plant Physiol. 154, 1403-1414

88. Lee, S. et al. (2016) Global regulation of plant immunity by histone lysine methyl transferases. Plant Cell 28, tpc.00012.2016

89. $\mathrm{Hu}$, M. et al. (2014) Histone H2B monoubiquitination is involved in regulating the dynamics of microtubules during the defense response to Verticillium dahliae toxins in Arabidopsis. Plant Physiol. 164, 1857-1865

90. Zou, B. et al. (2014) Monoubiquitination of histone 2B at the disease resistance gene locus regulates its expression and impacts immune responses in Arabidopsis. Plant Physiol. 165, 309-318

91. Dhawan, R. et al. (2009) HISTONE MONOUBIQUITINATION interacts with a subunit of the mediator complex and regulates defense against necrotrophic fungal pathogens in Arabidopsis. Plant Cell 21, 1000-1019

92. Walley, J.W. et al. (2008) The chromatin remodeler SPLAYED regulates specific stress signaling pathways. PLoS Pathog. 4, $1-8$

93. March-Díaz, R. et al. (2008) Histone H2A.Z. and homologues of components of the SWR1 complex are required to contro immunity in Arabidopsis. Plant J. 53, 475-487

94. Berriri, S. et al. (2016) SWR1 chromatin-remodeling complex subunits and H2A.Z. have non-overlapping functions in immunity and gene regulation in Arabidopsis. Mol. Plant 9, 10511065

95. Hilker, M. et al. (2016) Priming and memory of stress responses in organisms lacking a nervous system. Biol. Rev. 91, 11181133

96. Lämke, J. and Bäurle, I. (2017) Epigenetic and chromatin-based mechanisms in environmental stress adaptation and stress memory in plants. Genome Biol. 18, 124

97. Beckers, G.J.M. et al. (2009) Mitogen-activated protein kinases 3 and 6 are required for full Priming of stress responses in Arabidopsis thaliana. Plant Cell Online 21, 944-953

98. Slaughter, A. et al. (2012) Descendants of primed Arabidopsis plants exhibit resistance to biotic stress. Plant Physiol. 158 835-843

99. Luna, E. et al. (2012) Next-generation systemic acquired resistance. Plant Physiol. 158, 844-853

100. Rasmann, S. et al. (2012) Herbivory in the previous generation primes plants for enhanced insect resistance. Plant Physio. $158,854-863$

101. Ramírez-Carrasco, G. et al. (2017) Transgenerational defense priming for crop protection against plant pathogens: a hypothesis. Front. Plant Sci. 8, 1-8

102. Jaskiewicz, M. et al. (2011) Chromatin modification acts as a memory for systemic acquired resistance in the plant stress response. EMBO Rep. 12, 50-55

103. Mozgová, I. et al. (2015) Chromatin assembly factor CAF-1 represses priming of plant defence response genes. Nat. Plants 1,15127

104. van Hulten, M. et al. (2006) Costs and benefits of priming for defense in Arabidopsis. Proc. Natl. Acad. Sci. 103, 5602-560

105. Hamon, M.A. and Cossart, P. (2008) Histone modifications and chromatin remodeling during bacterial infections. Cell Host Microbe 4, 100-109

106. Clayton, A.L. and Mahadevan, L.C. (2003) MAP kinase-mediated phosphoacetylation of histone $\mathrm{H} 3$ and inducible gene regulation. FEBS Lett. 546, 51-58

107. Netea, M.G. et al. (2016) Trained immunity: a program of innate immune memory in health and disease. Science 352, 1-23 